“(C) 2015 IEEE. Personal use of this material is permitted. Permission from IEEE must be obtained for all other uses, in any current or future media, including reprinting/republishing this material for advertising or promotional purposes, creating new collective works, for resale or redistribution to servers or lists, or reuse of any copyrighted component of this work in other works." 


\section{Beamspace Time Reversal Maximum Likelihood Estimation for Microwave Breast Imaging}

\author{
Md. Delwar Hossain \\ Centre for Health Technologies, FEIT \\ University of Technology Sydney (UTS) \\ Sydney, NSW, Australia \\ Md.D.Hossain@student.uts.edu.au
}

\author{
Ananda Sanagavarapu Mohan \\ Centre for Health Technologies, FEIT \\ University of Technology Sydney (UTS) \\ Sydney, NSW, Australia \\ Ananda.Sanagavarapu@uts.edu.au
}

\begin{abstract}
We consider maximum likelihood based beamspace time reversal beamforming for breast cancer localization. We reduce the computational burden of maximum likelihood estimation through reduced dimensional beamspace processing. Beamspace processing also provides additional beamspace gain which contributes to suppress strong clutter effects. We collect multistatic scattering fields through FDTD simulation and further process it in beamspace for maximum likelihood based time reversal imaging. The imaging technique is used to localize a small tumor in a dense breast. It is observed that the proposed imaging technique can localize tumors unambiguously even in dense breast phantom.
\end{abstract}

\section{INTRODUCTION}

Maximum likelihood (ML) estimation achieves superior imaging performance due to inherent possession of scaling factor compared to time reversal imaging [1]. Appropriate scaling factor helps to provide balanced images to identify scatterers far away. This is a desired factor in microwave imaging for breast cancer detection since high tissue heterogeneity significantly attenuates and distorts tumor response [2-8]. But maximum likelihood estimation is computationally intensive. In order to reduce the computational burden we use beamspace transformation [8]. Beamspace processing also provides additional beamforming gain to further suppress the undesired clutter scattering effects. We use a cylindrical transceiver array to obtain multistatic response matrix for a dense breast where a tumor is embedded. The scattered field from the breast phantom is obtained through FDTD simulation. Beamspace transformation is applied to the received signals prior to time reversal based maximum likelihood estimation. The rest of the paper is organized as follows. We provide details about breast phantom and FDTD simulation and beamspace time reversal maximum likelihood imaging technique in section II. We provide results in section III followed conclusions in section IV.

\section{NUMERICAL SIMULATION AND IMAGING}

We have used FDTD simulation to compute the scattered field from the numerical breast phantom. The scattered field is further processed to obtain the tumor location.

\section{A. FDTD Simulation Using Breast Phantom}

We have obtained anatomically realistic breast phantom from UWCEM phantom repository [9]. A tumor with $8 \mathrm{~mm}$ maximum dimension is embedded into the phantom. The phantom is surrounded by a cylindrical array consisting of 115 elements. The cylindrical array is formed by 5 circular rings where each ring contains 23 elements. A differentiated Gaussian pulse of center frequency $3 \mathrm{GHz}$ with $3-\mathrm{dB}$ bandwidth of $3.5 \mathrm{GHz}$ is used as the excitation. The scattered field is collected along the coordinate axes at each array element. The grid size is set to $0.5 \mathrm{~mm}$ in all directions and PML boundary condition is used. The simulation set-up is shown in Fig. 1. The blue dots indicate the array elements locations. The breast tissue dielctric properties are computed using single pole debye parameters $[10,11]$. In order to reduce skin reflection the breast phantom and the array are immersed into a matching liquid.

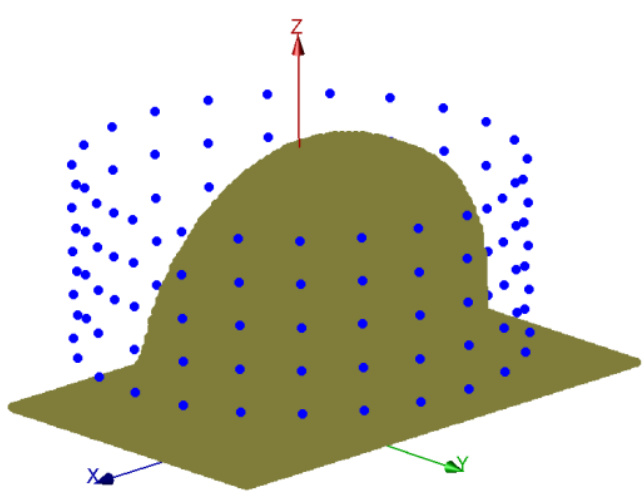

Fig. 1. Simulation set-up with breast phantom.

\section{B. Beamspace Time Reversal Maximum Likelihood Imaging}

The multistatic matrix resulting from multistatic illumination in the presence of additive white Gaussian noise can be expressed as follows [12].

$$
\overline{\overline{\mathbf{Y}}}=\overline{\overline{\mathcal{G}}} \overline{\overline{\mathbf{M}}}_{\varepsilon} \overline{\overline{\mathcal{G}}}^{T}+\sigma^{2} \overline{\overline{\mathbf{I}}}=\overline{\overline{\mathbf{K}}}+\sigma^{2} \overline{\overline{\mathbf{I}}}
$$

where, $\sigma^{2}$ is the noise variance. Now we use DFT based beamforming matrix $\overline{\overline{\mathbf{B}}}(3 N \times 3 F)$ for beamspace transformation.

$$
\overline{\overline{\mathbf{Y}}}_{B}=\overline{\overline{\mathbf{B}}}^{H} \overline{\overline{\mathbf{Y}}}=\overline{\overline{\mathbf{B}}} \overline{\overline{\mathbf{K}}}+\sigma^{2} \overline{\overline{\mathbf{B}}}^{H} \overline{\mathbf{I}}
$$


Here, $\overline{\overline{\mathbf{Y}}}_{B}$ has a reduced dimension of $3 F \times 3 N(F<N)$ where $N$ is the total number of array elements. We apply beamspace transformation in the receiving mode.

The maximum likelihood estimation of scattering strength can be written as

$$
\hat{\mathbf{\mathbf { x }},{\hat{m^{\varepsilon}}}^{\varepsilon}}=\underset{\mathbf{x}_{p}, \overline{\bar{m}}^{\varepsilon}}{\arg \min }\left\|\overline{\overline{\mathbf{B}}}^{H} \overline{\overline{\mathbf{Y}}}-\overline{\overline{\mathbf{B}}}^{H} \overline{\overline{\mathbf{K}}}\left(\mathbf{x}, \overline{\overline{m^{\varepsilon}}}\right)\right\|_{\mathrm{F}}^{2}
$$

Consequently the imaging function can be obtained as

$$
I\left(\mathbf{x}_{p}\right)=\frac{\left|\left(\overline{\overline{\mathbf{B}}}^{H} \overline{\overline{\mathbf{G}}}\right)^{H} \overline{\overline{\mathbf{Y}}}_{B} \overline{\overline{\mathbf{G}}}^{T}\right|^{2}}{\|\overline{\overline{\mathbf{B}}} \overline{\overline{\mathbf{G}}}\|_{F}^{4}\|\overline{\overline{\mathbf{G}}}\|_{F}^{4}}
$$

where, $\overline{\overline{\mathbf{G}}}$ is the dyadic green's function vector of background medium.

\section{RESULTS AND DISCUSSION}

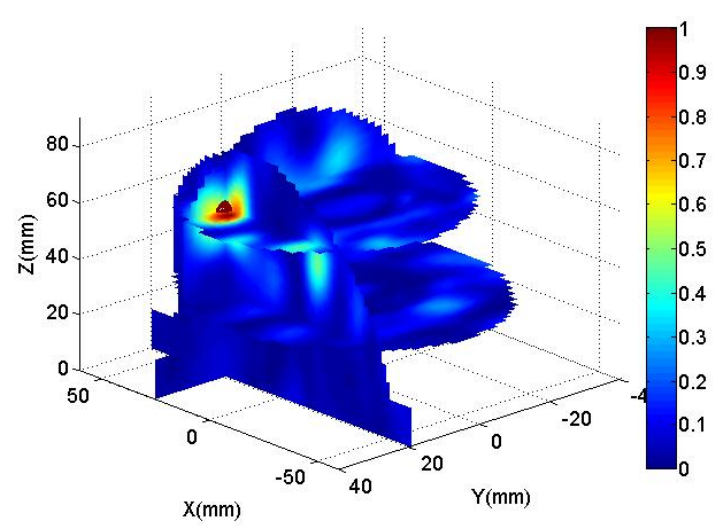

(a)

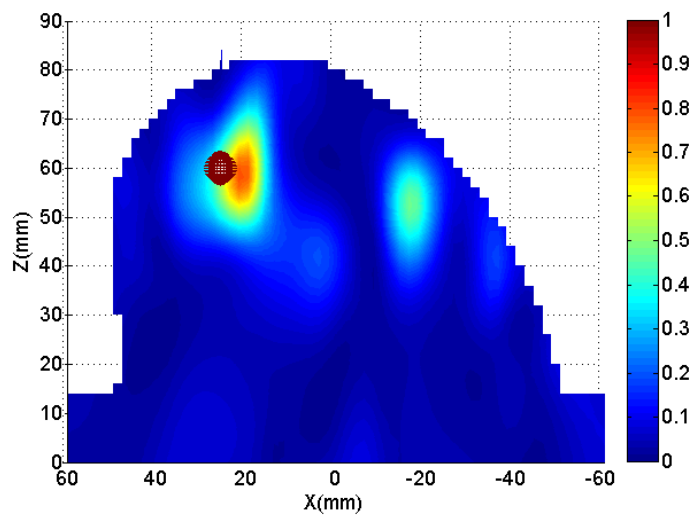

(b)

Fig. 2. (a) Cross section through estimated tumor location, (b) sagittal view.

The imaging result for a dense breast phantom is shown in

Fig. 2. The actual tumor location is indicated using the small red spherical inclusion. It is clearly observed that the highest intensity in the image very accurately coincides with the actual tumor location. We assumed an equivalent homogeneous background medium for time reversal imaging. Prior to beamforming skin artifact was removed using entropy based windowing technique [13].

\section{DISCUSSION}

The proposed imaging technique has successfully localized a tumor in a dense breast phantom. The tumor location is unambiguously identified while little effect of clutter is also observed. The computational load is also decreased through beamspace processing.

\section{REFERENCES}

[1] G. Shi and A. Nehorai, "A Relationship Between Time-Reversal Imaging and Maximum-Likelihood Scattering Estimation," IEEE Trans. Signal Process., vol. 55, pp. 4707-4711, 2007.

[2] J. D. Shea, B. D. Van Veen, and S. C. Hagness, "A TSVD Analysis of Microwave Inverse Scattering for Breast Imaging," IEEE Trans. Biomed. Eng., vol. 59, pp. 936-945, 2012.

[3] E. C. Fear, J. Bourqui, C. Curtis, D. Mew, B. Docktor, and C. Romano, "Microwave Breast Imaging With a Monostatic Radar-Based System: A Study of Application to Patients," IEEE Trans. Microw. Theory Tech., vol. 61, pp. 2119-2128, 2013.

[4] A. H. Golnabi, P. M. Meaney, and K. D. Paulsen, "Tomographic Microwave Imaging With Incorporated Prior Spatial Information," IEEE Trans. Microw. Theory Tech, vol. 61, pp. 2129-2136, 2013.

[5] E. Kirshin, B. Oreshkin, G. K. Zhu, M. Popovic, and M. Coates, "Microwave Radar and Microwave-Induced Thermoacoustics: DualModality Approach for Breast Cancer Detection IEEE Trans. Biomed. Eng., vol. 60, pp. 354-360, 2013.

[6] H. F. Abutarboush and M. Klemm, "Signal Selection for ContrastEnhanced UWB Microwave Radar Imaging With Inhomogeneous Breast Phantoms," IEEE Antennas Wireless Propag. Lett., IEEE, vol. 12, pp. 1408-1411, 2013.

[7] A. Shahzad, m. o'halloran, E. Jones, and M. Glavin, "Prefiltered Beamforming for Early-Stage Breast Cancer Detection," IEEE Antennas Wireless Propag. Lett, IEEE, vol. 12, pp. 500-503, 2013.

[8] M. D. Hossain, A. S. Mohan, and M. J. Abedin, "Beamspace TimeReversal Microwave Imaging for Breast Cancer Detection," IEEE Antennas Wireless Propag. Lett, vol. 12, pp. 241-244, 2013.

[9] Available: http://uwcem.ece.wisc.edu/MRIdatabase/index.htm

[10] M. Lazebnik, D. Popovic, L. McCartney, C. B. Watkins, M. a. J. Lindstrom, J. Harter, S. Sewall, T. Ogilvie, A. Magliocco, T. M. Breslin, W. Temple, D. a. Mew, J. H. Booske, M. i. Okoniewski, and S. C. H. agness, "A large-scale study of the ultrawideband microwave dielectric properties of normal, benign and malignant breast tissues obtained from cancer surgeries " Phys. Med. Biol., vol. 52, pp. 6093 6115, 2007.

[11] M. Lazebnik, M. Okoniewski, J. H. Booske, and S. C. Hagness, "Highly accurate debye models for normal and malignant breast tissue dielectric properties at microwave frequencies," IEEE Microw. Wireless Comp. Lett., vol. 17, pp. 822-824, 2007.

[12] E. Iakovleva, S. Gdoura, D. Lesselier, and G. Perrusson, "Multistatic Response Matrix of a 3-D Inclusion in Half Space and MUSIC Imaging," IEEE Trans. Antennas Propag., vol. 55, pp. 2598-2609, 2007.

[13] Z. Wanjun and F. Chin, "Entropy-Based Time Window for Artifact Removal in UWB Imaging of Breast Cancer Detection," IEEE Signal Process. Lett., vol. 13, pp. 585-588, 2006. 